\title{
Stakeholder dialogue on dilemmas at work as a workplace health promotion intervention including employees with a low SEP: a Responsive Evaluation
}

\author{
Hanneke van Heijster ${ }^{1 *}$, Jantien van Berkel ${ }^{1}$, Cécile R. L. Boot ${ }^{2}$, Tineke Abma ${ }^{3}$ and Emely de Vet ${ }^{1}$
}

\begin{abstract}
Background: The aim of this study was to evaluate the perceived changes of an innovative workplace health promotion intervention and evaluation. In this study, a bottom-up approach was taken to define the central themes and relevant outcomes of an intervention. These central themes and relevant outcomes of the intervention were defined together with stakeholders, including employees with a low socioeconomic position.

Methods: The intervention consisted of a series of structured stakeholder dialogues in which dilemmas around the - by employees defined —health themes were discussed. The intervention was implemented in a harbor service provider with approximately 400 employees. Over a two-year period, 57 participants engaged in eight dialogues of one hour. 15 interviews and six participant observations took place for the evaluation of the intervention.

Results: Together with the stakeholders, high workload and mental health were defined as central themes for the dialogue intervention in the male-dominated workplace. The dialogue intervention contributed to changes, on different levels: individual, team, and organization. Overall, the stakeholder dialogues advanced the understanding of factors contributing to high workload and mental health. In reply to this, several actions were taken on a organizational level.

Conclusions: Taking a bottom-up approach in WHP allows to understand the health issues that are important in the daily reality of employees with a low socioeconomic position. Through this understanding, workplace health promotion can become more suitable and relevant for employees with a low socioeconomic position.

Trial registration: Netherlands Trial Register (NRT): NL8051. Registration date: 28/09/2019, Retrospectively registered https://www.trialregister.nl
\end{abstract}

Keywords: Workers, Blue-collar, Health promotion, Worksite, Intervention, Participation, Participatory, Co-creation

*Correspondence: hanneke.vanheijster@wur.nl

${ }^{1}$ Department of Social Sciences, Chair Group Consumption \& Healthy Lifestyles, Wageningen University \& Research, Hollandseweg 1, 6706 KN Wageningen, Netherlands

Full list of author information is available at the end of the article

\begin{abstract}
Background
Significant health inequalities between individuals with low a socioeconomic position (SEP) and a high SEP exist in most Western-European countries [1,2]. Life expectancy of individuals with a low SEP can be up to 10 years shorter than of individuals with a high SEP [3]. Also, individuals with a low SEP live between 10 to 23 years shorter in good health [4]. Workplace health promotion (WHP)
\end{abstract}


is considered promising to improve health of employees with a low SEP. The workplace gives access to part of the generally hard to reach low SEP population, as half is employed [5] and employees spend much time of their lives at work [6, 7]. Also, the workplace offers a physical and social infrastructure necessary for health promotion [8]. Therefore, WHP has the potential to contribute to the reduction of health inequalities.

However, it is doubtful if WHP in its current form does contribute to the reduction of health inequalities. Recent Individual Participant Data (IPD) meta-analyses on in total 15 Dutch WHP intervention studies, showed no effects on BMI [9]-except from small effects for highrisk groups under specific conditions-and no effects on lifestyle behaviors [10] of employees with both low and high SEP, and no effects on self-perceived health of employees with a low SEP [11]. A meta-analysis including mainly intervention studies from the US found some evidence that physical activity interventions at work may be effective in reducing health inequalities, but the evidence base was small and of low quality [12].

Three possible underlying reasons for the disappointing effects of current WHP have been described before [13]. First, the lack of acknowledgement of diverging values and interests of the many stakeholders involved in WHP, such as employers, employees, intervention providers, research and knowledge institutes and insurance companies [14]. These perspectives may often be competing, possibly affecting the effects and relevance of WHP [15]. Second, WHP evokes ethical questions. For example about who is responsible for employees' health and what this responsibility entails [16], whether and to which extent interference in privacy of employees is acceptable, and about voluntariness of participation while power dependencies between employer and employee in the workplace exist $[14,16,17]$. Third, employees with a low SEP generally lack voice in the design and evaluation of WHP [14], being rather researched upon, than with [15]. Involving employees in WHP-those with first-hand experience of the particular workplace-may increase its relevance [15]. This first-hand experience is especially relevant when it comes to employees with a low SEP, as insight in how to target their health effectively considering their lifeworld, is scarce [18]. WHP may be more suitable when deliberate attention is paid to the aforementioned underlying reasons.

This study involves an innovative WHP intervention and evaluation in which the underlying reasons for previous limited effects are taken into account. A bottom-up approach is taken to define the central themes for the intervention, where special attention is paid to involve employees with a low SEP through a participatory approach to evaluation: Responsive Evaluation [19].
In Responsive Evaluation stakeholders are active partners in defining central themes and relevant research changes [20]. To date, it has been more common in WHP that central themes and outcomes of an intervention are defined by the researchers [14]. Also, being involved in defining central themes may enhance the relevance of WHP for employees with a low SEP [20], thereby offering a possible solution for low participation of employees with a low SEP [21-23].

The intervention consists of a series of structured stakeholder dialogues, in which participants discuss dilemmas around the central health themes. Participants bring in experiences from their daily experiences [13]. Rather than an educative or counseling component, the experiences of participants are central in the intervention. By bringing together and confronting a variety of perspectives in the dialogues, a learning process can emerge and shared insights can be gained. This learning process can take place at various levels, including the case, individual, team and organizational level [24].

The aim of this study is to evaluate stakeholder dialogue as an intervention for WHP in two ways. First, together with stakeholders, themes for and the desired outcomes of the dialogues will be defined. Second, it will be evaluated with stakeholders whether and which changes are perceived during and after the stakeholder dialogue.

\section{Methods}

An extensive description of methods was provided in the Study Protocol of this study published elsewhere [13].

\section{Setting}

During two years, the study was conducted in a harbor service provider (industrial sector) with approximately 400 employees, in The Netherlands.

\section{Design}

The intervention was evaluated through Responsive Evaluation, a participatory form of evaluation [20]. Responsive evaluation constitutes a iterative research process in which data collection and analysis partly overlap [25]. More details about this form of evaluation are described elsewhere [13]. Methods in the two-year evaluation were interviews, participant observations and HRM-data (Fig. 1). These methods were used for two purposes. First, to define the themes and relevant outcomes for the stakeholder dialogues. Second, to evaluate changes after or during the stakeholder dialogues, as perceived by the stakeholders.

\section{Intervention}

The aim was to perform five to six stakeholder dialogues per year [13]. Base for the stakeholder dialogues 


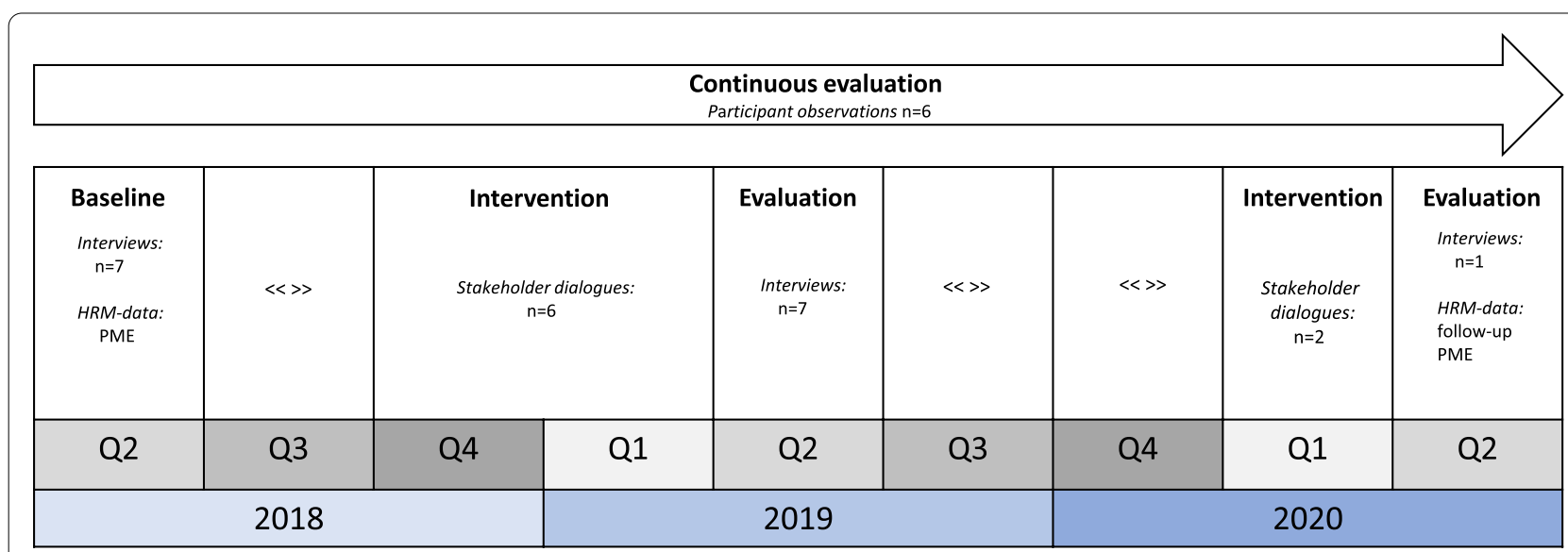

Fig. 1 Schematic overview of the Responsive Evaluation and Intervention [PME = Periodic Medical Evaluation. $<<>>$ indicates that there was no research activity during this period]

was a form of moral case deliberation (MCD), namely the Dilemma-method [26]. In short, in a dialogue according to the Dilemma-method, participants bring in dilemmas they face in their daily work (e.g. should I as for help if I'm too busy, while I think this is not accepted in the culture of this organization?). The dialogue facilitator helps participants to look at the different perspectives (interests, values, norms) on this dilemma, for example the perspective of the employee, employer, client and colleagues. After evaluating the different options for action in this dilemma, all participants formulate what they would to in the situation. Differences between solutions are discussed according to the rules of dialogue (e.g. postpone judgments). Also, the participants deliberate about the individual and/ or organizational actions that are necessary to act in the desired manner. The emphasis on mutual learning among participants and the focus on the ethical dimensions of issues and experiences of participants differs MCD from similar methods such as focus groups and health circles [27]. This form was considered best suitable for the purpose of the project [13].

The Dilemma-method was adapted in various ways to make it suitable for the work setting and its employees [13]. To date, the Dilemma-method has not been used specifically for workplace health promotion and with employees with a low SEP. The method is traditionally used for health care professionals to deliberate about dilemmas they encounter in their daily care-practices [28]. Several adaptations were made to make the dialogue method feasible for the setting of this study. These adaptations are explained in Additional file 1, which includes the dialogue guide and also describes the adaptations made throughout the evaluation based on advancing insights.

\section{Sampling and recruitment}

A proportionate universalism approach was taken to recruit participants [29]. This means that all stakeholders were eligible to participate. However, special attention was paid to include employees with a low SEP, as they generally participate less in WHP interventions. Employees with a higher SEP were not excluded from the intervention, because the intervention was based on the rationale that context changes would benefit employees with low SEP the most. In other words, the entire organisation was eligible for participation as they constitute the (social) context.

Stakeholders could participate in the intervention (stakeholder dialogues) and evaluation (interviews, participant observations), but also to one of both. Participants were recruited for the intervention and evaluation via contact persons in the organization. Participation was based on willingness to participate. Operational employees were asked to indicate with whom they would feel comfortable enough to have the dialogues with, as a prerequisite for a safe communication climate. Mixed groups with employees and direct supervisors from different departments were preferred.

Participants received an email from the researchers $(\mathrm{HvH}, \mathrm{JvB})$ with information about the dialogue (duration, location, aim) and explanation for preparation. Participants were invited to think of a dilemma related to the central themes, that were defined earlier in the Responsive Evaluation. The aim was to have six to twelve participants per dialogue.

\section{Participants}

In total there were 16 participants in 15 interviews. Participants worked at various departments in the organization (management or support staff (7), supervisors (4), and operational employees (5)). Participant observations 
were performed at two operational departments during three toolbox sessions, that were attended by operational employees. In the dialogues, 57 participants participated over eight dialogues. The number of participants in the dialogues ranged from four to 11 . The majority was male (90\%). $20.5 \%$ of all participants were operational staff with a low SEP (low educational requirement). Educational requirement was defined by the researchers and was based on the educational level required for the job. Participants were not asked for their educational level to avoid stigmatization. Educational requirement was used as an estimation. The group composition of the dialogues was determined based on the preferences of operational employees [25].

In the first year, six dialogues took place, and two in the second year. The lower number of dialogues in the second year was the result of 1) a merge of locations, reducing the amount of locations where dialogues could be performed, 2) saturation in terms of central themes and new learnings in the dialogues.

\section{Data collection}

Central themes and desired changes of the intervention were defined with stakeholders and continuously monitored throughout the evaluation. Perceived changes of the intervention were evaluated both at fixed moments (i.e. after one and two years) and continuously (Fig. 1).

Semi-structured interviews and participant observations were used to define the central themes and desired changes of the stakeholder dialogues according to the stakeholders (Fig. 1, baseline), and to evaluate the perceived changes during and after the stakeholder dialogues (Fig. 1, evaluation 1 and 2). Topics of the interviews at baseline and at evaluation moments are described in the Study protocol [13]. Periodic Medical Examinations (PME) were used as an additional source of data to verify the scope of the central themes throughout the organization. In addition, all forms of communication with stakeholders (e-mails, logs of phone calls) served as an additional source of data for evaluation.

\section{Data analysis}

Thematic content analysis was performed to analyze data from interviews and dialogues. Analysis about the relevant themes for and desired outcomes of the stakeholder dialogues (baseline) and the perceived changes (evaluation 1 and 2) proceeded inductively. Perceived changes were categorized into changes on four levels, namely case, individual, team, organizational level. These levels were based on the four aims of MCD, the type of stakeholder dialogue used in this study [30], and the EURO-MCD classification [24]. All interviews were first individually coded. Subsequently, comparisons and differences between interviews were made. Atlas. ti 9 Windows was used for qualitative analysis (Coding trees can be found in the Additional file 2). Analysis of the stakeholder dialogues also proceeded inductively, and perceived changes of the dialogues were also categorized into the aforementioned four levels.

\section{Quality measures}

Several quality procedures for qualitative research were taken, as recommended by Frambach et al [31]. These measures are described in the Study Protocol of this study [13] and reflected upon in the discussion.

More details about the methods can be found in the Study Protocol published elsewhere [13].

\section{Results}

The results are presented in two parts, following the research aims. Part I describes the central themes that were defined with the stakeholders. Part II describes the desired changes before the intervention, and the perceived changes during and after the intervention.

\section{Part 1 - Central themes}

Two relevant health related themes stood out throughout the entire evaluation period: high workload and mental health.

\section{High workload}

This reoccurring theme was often attributed to the unpredictable nature of the work, leading to high peaks and insufficient numbers of personnel. For operational employees, high physical job demands (working with dangerous goods) and mental job demands (multitasking, prioritizing on the spot) also contributed to perceiving a high workload. According to employees, high workload influenced health by disturbing the work-life balance, working less safe, reduced job satisfaction or mental pressure of the potential consequences of mistakes and unsafe working (e.g. losing clients). Working less safe (not fully according to the safety regulations) was especially a concern for younger employees with little experience according to supervisors:

\section{"Those young boys that just got employed, you have to tell them: dude, calm down. They think: how can I do this as quickly as possible? And then they start run- ning and flying, but you shouldn't do that. Because with doing that in this job, you risk your safety. They are like oh I forgot to put my helmet because I was too busy."-Supervisor, baseline interview 1.}

The consequences of mistakes, i.e. not following the safety rules or other mistakes because of a perceived high workload, could be far-reaching. Employees seemed to 
Table 1 Topics and dilemmas in the dialogue sessions

\begin{tabular}{lll}
\hline Session & Topic & Dilemma discussed (brought in by participants) \\
\hline 1 & Balance between working fast and safe & Being a good employee and colleague or working safe and healthy \\
2 & & Protecting reputation or protecting health \\
3 & & Being a good employee and colleague or working safe and healthy \\
4 & Discussing (health and safety) issues with colleagues and & Speaking up or being a good employee and colleague \\
5 & supervisors & Speaking up or being a good employee and colleague \\
6 & Discussing burn-out with colleagues and supervisors & Own responsibility or strict regulations \\
8 & & Help a colleague with burn-out symptoms or protect his reputation \\
\hline
\end{tabular}

have a feeling of responsibility regarding the reputation of the organization.

"We are talking about cargos of over hundred million sometimes. If you make a mistake because you are mentally out of the world for a moment, yes then..."... "If something happens at our plant, [name organization] will take the blame." - Operational employee, baseline interview 2.

\section{Mental health}

Employees and management noticed an increase of colleagues that were absent because of a burn-out or stress symptoms. Periodic Medical Evaluations (PME) that were performed during the course of the project (June 2018 \& April 2019), showed that employees with a low SEP scored below national averages on aspects of mental health such as work engagement and above on burn-out and stress. Masculine norms were reported as a contributing factor to burn-out. Keeping the image of being a strong worker and preferable not showing vulnerability impeded employees to speak up at an early stage, even though it was mentioned that the organization is helpful when someone has mental complaints,

"They are, after all, a bit young guys, uh yes how do you say that politely? Hard working people, you see? It is really what you see in the news, the Rotterdam mentality." - Supervisor, baseline interview 1.

"We are here with kind of tough men and it's not cool of course to say, yes, things are not great at home or I don't feel so good..." "Usually we see it when it's too late. You notice that people are mentally absent, and then all of a sudden they have a burn-out." - Operational employee - baseline interview 2.

\section{Topics for moral case deliberation}

Based on the overarching themes high workload and mental health, topics for the dialogues were formulated.
The researchers searched for concrete examples of the formulated central themes in the data. The topics were discussed with the contact person of the organization. Table 1 presents an overview of the topics and dilemmas of each session is presented.

\section{Part 2 - Perceived changes}

\section{Desired changes before intervention}

Stakeholders were asked what they considered relevant changes of the intervention [20]. Interviewees were interested in learnings, either non-specified (i.e. cross pollination about how other departments deal with problems), or more specified (e.g. about how employees in other departments experienced the high workload). In addition, employees from various departments indicated that the dialogues could help defining shared experiences and/or structural issues that require improvement. The dialogues could be a means to jointly come up with ideas for improvement for the decision makers, thereby creating bottom-up support:

"I mean, if everyone says the same thing.. then the organization has something to work on." - Operational employee, baseline interview 6 .

The management team was also interested in learnings for improvement. For example, they indicated that it was relevant for them to learn how to could communicate more effectively with the 'shop-floor'.

\section{Perceived relevant changes after intervention}

Changes were perceived on all four levels (case, individual, team, organizational). Table 2 presents an overview of all perceived changes with a thick description of the context showing the relevance of the changes for the stakeholders.

Below, one change per level is described in detail. We selected changes that were not a single event, such as the purchase of a safety means, but were assumed to have a longer-term duration (e.g. perceived enhanced mutual understanding). 


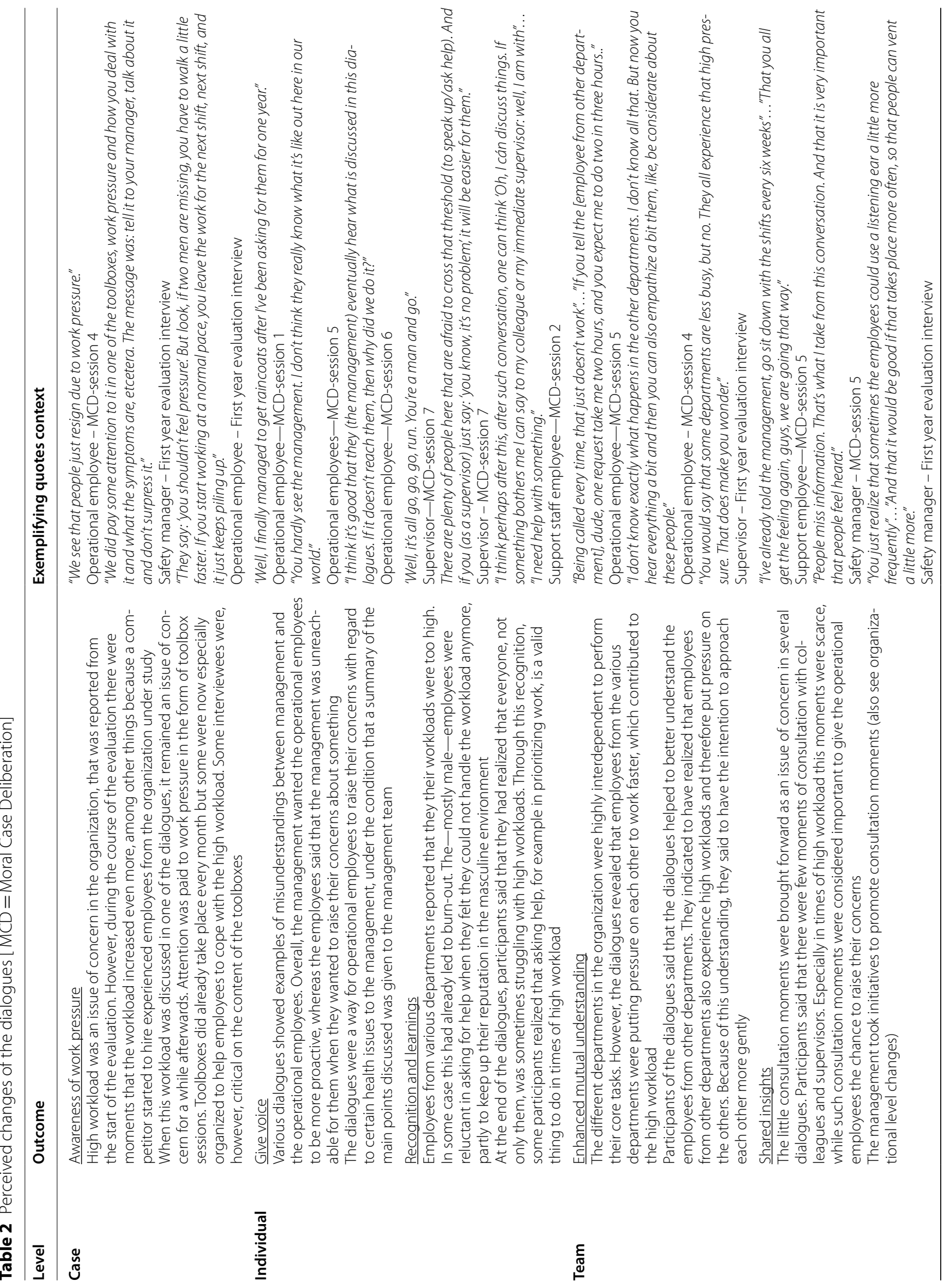




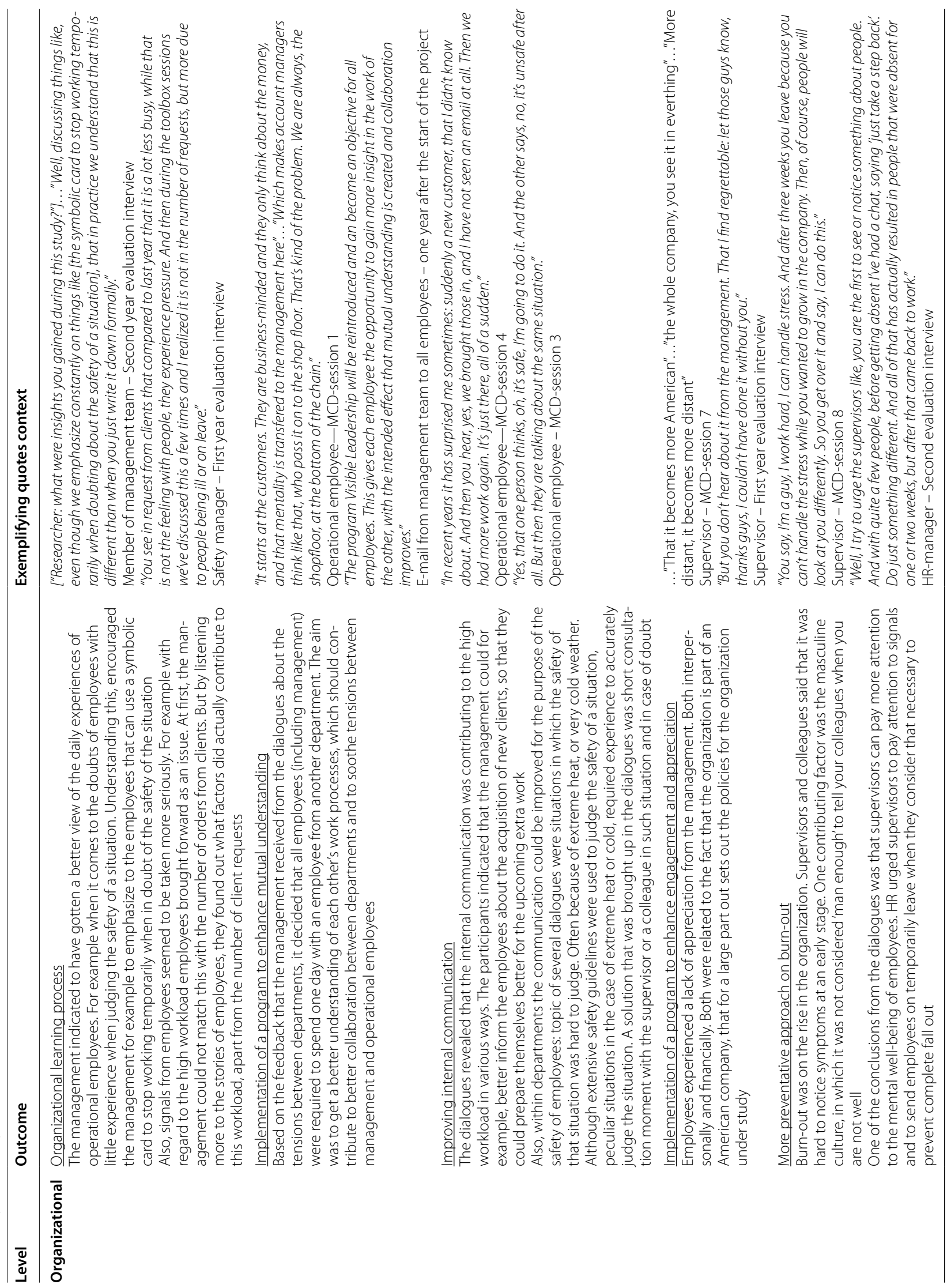




\section{Case level}

Agenda setting Some dialogues led to follow-up discussions about topics similar to the ones discussed in the dialogue. These follow up discussions were initiated by the organization, rather than by the researchers. For example, after a dialogue in which the peak of workload at that moment was discussed, 'toolbox' sessions were organized about the experience of high workload. In these sessions it was discussed how to prioritize tasks and how to deal psychologically with high workloads.

One operational employee that participated in a dialogue mentioned in the evaluation that the effort to reduce (the experience of) high workloads increased strongly directly after the dialogue. Yet, it was emphasized that this attention decreased after some time when the workload increased again. Nevertheless, changes on other levels occurred that were also related to the experience of high workload.

\section{Individual level}

Recognition and learnings The dialogues led to recognition of issues for the participants of the dialogues. Participants realized that colleagues, either from the same of from different departments, experienced similar issues, such as the high workload. It was reassuring for participants of various departments to realize that their department was not the only one experiencing high workload, but that it is a companywide issue. Also, the dialogues revealed that the prevailing masculine norms like being a strong worker, preferably not showing vulnerability, prevent employees from asking help. Participants indicated that they realized during the dialogue that asking for help in times of very high workload is a legitimate thing to do. Participants also realized that it may also be helpful for other employees not participating in the dialogues to know that it is not a problem to ask for help and that this should be communicated more actively.

\section{Team level}

Perceived enhanced mutual understanding Participants mentioned that the sessions contributed to enhancing the mutual understanding between departments. Tensions between departments, that are strongly interdependent for their core activities, was a factor that contributed to the experience of high workloads. Participants of the dialogues indicated that they sometimes got surprised by the perspectives of employees from other departments. Insight in their perspectives and working conditions enhanced understanding for certain situations that contributed to the experience of high workload. Moreover, the organization implemented an exchange program between departments to enhance the mutual understanding further.

\section{Organizational level}

Organizational learning process The dialogues helped the management to better understand the underlying factors of the central themes, high workload and mental health. From the perspective of the management, there were no signals about an increase in workload; there was no increase in requests from clients. However, during the course of the project, members of the management team started to learn via the dialogues what were the underlying reasons for the perception of high workload. Insight in these reasons, such as the sometimes compelling communication and tensions between departments, allowed the management to take targeted actions. For example, the management implemented a communication training for supervisors to promote respectful communication and proactiveness of employees in order to involve them more in daily practice. Other actions that were taken by the management were the implementation of an exchange program with the aim to learn about each other's work, initiatives to enhance the engagement of employees in organizational developments and stimulating a more preventative approach on burn-out by making supervisors aware that they are the ones that can signal symptoms at an early stage.

\section{Discussion}

This paper describes the evaluation of an innovative WHP study in which central themes for and desired changes of the intervention were defined together with employees with a low SEP and other stakeholders. High workload and mental health turned out to be wide-spread issues in the organization under study. In the stakeholder dialogues, participants shared examples of their own experiences with these themes. This initiated a learning process in the organization, in which the management gained more understanding of the factors playing a role in mental health and high workload. In reply to this, several actions were implemented on the organizational level.

An unique future of this study was the active role employees played in defining the central themes of the intervention. Participatory research designs are not yet common in the field of WHP, although they have been 
recommended [11, 32, 33] and explored [34, 35]. In a classification of the degree of participation in participatory research from Fetterman [36], this study could be classified in 'Collaborative Evaluation'. There was ongoing engagement between researchers and stakeholders. However, the researchers remained in charge of some of the main decisions, such as the method of the intervention, as well as for the methods of evaluation, although they were adjusted to the work setting under study. In the classification of Fetterman the Collaborative Evaluation is the lightest form of participation. Nonetheless, on the ladder of participation of Arnstein [37], this study could be placed on step six 'Partnership' (the ladder includes step one to eight, eight being the highest degree of participation).

The stakeholder dialogues are expected to have contributed to health of employees with a low SEP in two ways. First, through the actions that followed from the dialogues. Most of the actions related to improvements in the work context. It has been shown that working conditions contribute as much and sometimes more than healthy behaviors to health of employees with a low SEP $[38,39]$. Second, participants of the dialogues reported learnings after participating. A concrete example being the insight that asking for help in busy times can be considered a legitimate thing to do. Employees may have profited from this learning in situations in which they had high workloads.

Next to the actions, the group composition in the dialogues-mostly homogeneous groups in the sense of dependency relations-may have been advantageous to employees and social relations in the organization. Although one of the reasons to study a stakeholder dialogue as an intervention was the variety of stakeholders involved in WHP, mainly one stakeholder group participated in the dialogues, namely employees, although from different departments and with a variety of functions, aligned with their preferences [13]. Homogeneous groups may be advantageous in hierarchical organizations - such as the organization under study-because they allow for so-called 'enclave deliberation', in which like-minded people discuss topics together. This has been shown to enhance self-efficacy and interpersonal trust [40] and might as well have established a safe communication climate [25]. It may also help to deal with power differences between groups and forestalls domination by established groups [41]. However, which group composition is favorable depends on the power relations in the organization where the intervention is implemented.

It should be recognized that there were several favorable circumstances for Responsive Evaluation and stakeholder dialogue. First, the organization under study allowed that the dialogues took place during working time. This probably enhanced the willingness of employees to participate. Second, the organization was open for feedback, a requisite for participatory research to succeed [42]. Possibly, this openness was related to organization's focus on safety and the associated continuous attention for improvement. However, the first dialogue yielded a lot of response. Some participants expressed their frustration about other participants who, in their eyes, used the dialogues as a platform to 'just' express their frustrations without being constructive. The turmoil evoked worries about the upcoming dialogues, also at the higher level management. In the following dialogues, the researchers paid more attention to the underlying concerns of the expressed frustrations and on what could be helpful to these concerns. Similar strong responses on the dialogues did not occur again. In fact, the strong reactions on the first dialogue were in hindsight perceived as a sign that employees should be heard more regularly.

Also, the gender of the researchers (both women) may have played a role in how health issues were discussed in the dialogues. The researchers noticed that participants were spoke openly about issues such as mental health and high workload in dialogues and interviews, while the same participants mentioned that there was a lack of openness about these issues because of the prevailing masculine norms. Possibly, the participants felt comfortable about discussing the themes because they perceived the female researchers as 'empathic listeners' [43], and being women, 'allowed' to care and ask questions about health [44]. Also, the researchers paid explicit attention to their language. They based their language on how employees themselves talked about mental health and high workload in participant observations and interviews. For example, participants never used the word 'stress', but used 'high workload'. This may have contributed to a safe communication climate.

\section{Strengths and limitations}

A strength of this study was the variety of data sources (data triangulation [31]) used to identify and monitor the central themes. The combination of interviews, participatory observations, PME-data and the dialogues allowed to get an varied view of the issues and the factors related to it. Also, the interpretation of the results took place in consultation with the participants (member check (30)). After each interview and group dialogue, the participants received a short summary made by the researchers. Participants could adapt or approve these summaries, thereby serving as a member check to verify the correctness of the interpretations of the researchers. After approval of the participants, the summaries were used to inform the higher management about the dialogues. This 
feedback loop was strongly valued by the participants; without informing the decision makers there would not be no further impact on their daily working life.

The type of evidence provided with this study can be considered a limitation. The perceived changes were identified by means of qualitative data. No statistical evidence was gathered about the effects of the intervention This impeded comparison of findings of various studies in a statistical manner. Fortunately, the qualitative data were informative on the experiences and perceived changes of the dialogues. The qualitative findings can only be transferred to similar settings (male-dominated large organizations ( $>250$ employees)), through the 'thick description' of the work setting given in the results [42]. The thick description of the work context, stakeholders and circumstances, allows other researchers or professionals to relate the findings to the context of their interest. Another limitation is that the initiated actions on an organizational level, only started to take place after one year. Therefore, it was not evaluated how employees appreciated and were affected by these actions on the longer term.

\section{Implications for practice and research}

Employers can learn from this study that actively asking employees to share health related issues from their daily experience can lead to shared insights about the factors contributing and withholding to their health. New interventions can take from this study that regarding employees as partners in WHP allows to understand the health issues relevant to their daily reality. Through this understanding WHP can be better adapted to the lifeworld of employees with a low SEP.

\section{Conclusion}

The Responsive Evaluation and stakeholder dialogue initiated and facilitated a learning process in an organization around central health themes, high workload and mental health. Although the perceived changes identified in this study are specific for the context under study, other organizations can learn what the result of dialogue with employees can be for their own WHP. Researchers, intervention providers and other stakeholders can take from this study that employees with a low SEP can be reached in WHP by involving them in in the intervention and evaluation. Also, it allows to understand the health issues that are relevant for employees, thereby making WHP more suitable for employees with a low SEP.

\section{Abbreviations}

SEP: Socioeconomic position; WHP: Workplace health promotion; MCD: Moral Case Deliberation.

\section{Supplementary Information}

The online version contains supplementary material available at https://doi. org/10.1186/s12889-022-12802-z.

Additional file 1. Guideline stakeholder dialogue

Additional file 2. Coding schemes

\section{Acknowledgements}

We would like to thank the organization for participating in this study and all the participants in that were involved in the intervention and evaluation.

\section{Authors contributions}

All authors provided intellectual input for the design of the study. $\mathrm{HvH}$ and JvB were responsible for the data collection and data analysis. $\mathrm{HvH}$ drafted this paper, in collaboration with JVB, and all other authors contributed to the further writing of the manuscript and read and approved the final version of the manuscript.

\section{Funding}

The study was funded by The Netherlands Organization for Health Research and Development (ZonMw; grant number 53-1001-411). The funding source did not play a role in the research or preparation of the article.

Availability of data and materials

Not applicable.

\section{Declarations}

Ethics approval and consent to participate

This study was approved by the Social Ethics Committee, on behalf of Wageningen University and Research. Written informed consent was obtained from all subjects for the research activities. For participant observation, oral informed consent was obtained. Information was presented on a literacy level that was considered acceptable for all employees (B1 literacy level). All methods were carried out in accordance with relevant guidelines and regulations.

\section{Competing interests}

The authors declare that they have no competing interests.

\section{Author details}

${ }^{1}$ Department of Social Sciences, Chair Group Consumption \& Healthy Lifestyles, Wageningen University \& Research, Hollandseweg 1, 6706 KN Wageningen, Netherlands. ${ }^{2}$ Department of Public and Occupational Health, Amsterdam Public Health Research Institute, Amsterdam UMC, VU University, Amsterdam, Netherlands. ${ }^{3}$ Department Public Health, Leiden University Medical Centre, Leiden University, Leiden, Netherlands.

Received: 10 September 2021 Accepted: 11 February 2022

Published online: 28 February 2022

\section{References}

1. Mackenbach JP, Stirbu I, Roskam AJR, et al. Socioeconomic inequalities in health in 22 European countries. N Engl J Med. 2008;358:2468-81.

2. Mäki N, Martikainen P, Eikemo T, et al. Educational differences in disabilityfree life expectancy: A comparative study of long-standing activity limitation in eight European countries. Soc Sci Med. 2013;94:1-8.

3. Kunst AE, Bos V, Lahelma E, et al. Trends in socioeconomic inequalities in self-assessed health in 10 European countries. Int J Epidemiol. Epub ahead of print 2005. DOI: https://doi.org/10.1093/ije/dyh342.

4. OECD. Health at a Glance: Europe 2016: State of Health in the EUtle. Paris: OECD Publishing, Paris, 2016. Epub ahead of print 2016. https://doi.org/ 10.1787/9789264265592-en.

5. CBS. Arbeidsdeelname naar onderwijsniveau, https://www.cbs.nl/nl-nl/ visualisaties/dashboard-arbeidsmarkt/werkenden/arbeidsdeelnamenaar-onderwijsniveau. Accessed 27 May 2019. 
6. Golden SD, Earp JAL. Social Ecological Approaches to Individuals and Their Contexts: Twenty Years of Health Education \& Behavior Health Promotion Interventions. Heal Educ Behav. 2012:39:364-72.

7. Meng L, Wolff MB, Mattick KA, et al. Strategies for Worksite Health Interventions to Employees with Elevated Risk of Chronic Diseases. Saf Health Work. 2017;8:117-29.

8. Baron SL, Beard S, Davis LK, et al. Promoting integrated approaches to reducing health inequities among low-income workers: Applying a social ecological framework. Am J Ind Med. 2014:57:539-56.

9. Robroek SJ, Oude Hengel KM, van der Beek AJ, et al. Socio-economic inequalities in the effectiveness of workplace health promotion programmes on body mass index: An individual participant data meta-analysis. Obes Rev. 2020. https://doi.org/10.1111/obr.13101.

10. Suzan JW Robroek, Karen M Oude Hengel, Allard J van der Beek, Cécile RL Boot, Frank J van Lenthe, Alex Burdorf PC. Socioeconomic inequalities in the effectiveness of workplace health promotion programmes on body mass index: an individual participant data meta-analysis. Obes Rev. 2020;21(11):e13101. https://doi.org/10.1111/obr.13101.

11. van Heijster $H$, Boot CR, Robroek SJ, et al. The effectiveness of workplace health promotion programs on self-perceived health of employees with a low socioeconomic position: An individual participant data metaanalysis. SSM - Popul Heal. 2021;13:100743.

12. Cairns JM, Bambra C, Hillier-Brown FC, et al. Weighing up the evidence: A systematic review of the effectiveness of workplace interventions to tackle socio-economic inequalities in obesity. J Public Heal (United Kingdom). 2015;37:659-70.

13. Van Heijster $H$, Van Berkel J, Abma T, et al. Responsive evaluation of stakeholder dialogue as a worksite health promotion intervention to contribute to the reduction of SEP related health inequalities: A study protocol. BMC Health Serv Res. 2020;20:1-9.

14. Meershoek A, Horstman K. Creating a market in workplace health promotion: the performative role of public health sciences and technologies. Crit Public Health. 2016;26:269-80.

15. Allender S, Colquhoun D, Kelly P. Competing discourses of workplace health. Health (Irvine Calif). 2006;10(1):75-93.

16. Van Berkel J, Meershoek A, Janssens RM, et al. Ethical considerations of worksite health promotion: An exploration of stakeholders'views. BMC Public Health. 2014:14:1-10.

17. Ten HM, Van Der Heide A, Mackenbach JP, et al. An ethical framework for the prevention of overweight and obesity: A tool for thinking through a programme's ethical aspects. Eur J Public Health. 2013;23:299-305.

18. Medisch U, Groningen C, Tilburg U Van. Kennissynthese Werk(en) is Gezond, http://www.nvka.nl/resources/Kennissynthese_Werk_en_is_gezond_ eindrapport.pdf (2016).

19. Abma TA. Responsive evaluation: Its meaning and special contribution to health promotion. Eval Program Plann. 2005;28:279-89.

20. Abma, Tineke. Banks, Sarah. Cook, Tina. Dias, Sónia. Madsen, Wendy. Springett, Jane. Wright MT. Participatory research for health and social well-being. 1st ed. Springer Nature Switzerland AG, 2019.

21. Stiehl E, Shivaprakash N, Thatcher E, et al. Worksite Health Promotion for Low-Wage Workers: A Scoping Literature Review. Am J Heal Promot. 2018;32:359-73.

22. Magnée T, Burdorf A, Brug J, et al. Equity-specific effects of 26 dutch obesity-related lifestyle interventions. Am J Prev Med. 2013;44:e57-66.

23. White M, Adams J, Heywood P. How and why do interventions that increase health overall widen inequalities within populations? In: Babones S, editor. Health, inequality and society. Bristol: Policy Press; 2009. p. 64-81.

24. Svantesson M, Karlsson J, Boitte P, et al. Outcomes of Moral Case Deliberation - The development of an evaluation instrument for clinical ethics support (the Euro-MCD). BMC Med Ethics. 2014;15. https://doi.org/10. 1186/1472-6939-15-30.

25. Abma, T.A. Widdershoven GAM. Responsieve methodologie. Den Haag: Uitgeverij LEMMA; 2006

26. Van Dartel HMB. In gesprek blijven over goede zorg, overlegmethoden voor moreel beraad. 3rd ed. Amsterdam: Boom Uitgevers Amsterdam; 2018.

27. Aust B, Ducki A. Comprehensive health promotion interventions at the workplace: Experiences with health circles in Germany. J Occup Health Psychol. 2004;9:258-70.
28. van der Dam S, Abma TA, Molewijk AC, et al. Organizing moral case deliberation experiences in two dutch nursing homes. Nurs Ethics. 2011:18:327-40.

29. Caan W. Fair society, healthy lives: Timing is everything. BMJ. 2010:340:494.

30. Van Dartel H, Molewijk B. In gesprek blijven over goede zorg - overlegmethoden voor moreel beraad. Amsterdam: Uitgeverij Boom; 2014

31. Frambach JM, Van Der VCPM, Dunning SJ. AM Last Page AM Last Page : Quality Criteria in Qualitative and Quantitative Research. Acad Med. 2013;88:552.

32. Van De Ven D, Robroek SJW, Burdorf A. Are workplace health promotion programmes effective for all socioeconomic groups? A systematic review. Occup Environ Med 2020; 1-8.

33. WHO. WHO /Workplace health promotion. https://www.who.int/occup ational_health/topics/workplace/en/index1.html. Accessed 9 Sept 2020.

34. van den Berge $M$, Hulsegge $G$, van der Molen $H F$, et al. Adapting citizen science to improve health in an occupational setting: Preliminary results of a qualitative study. Int J Environ Res Public Health. 2020;17:1-19.

35. McVicar A, Munn-Giddings C, Seebohm P. Workplace stress interventions using participatory action research designs. Int J Work Heal Manag. 2013;6:18-37.

36. Fetterman D, Rodríguez-Campos L, Wandersman A, et al. Collaborative, Participatory, and Empowerment Evaluation: Building a Strong Conceptual Foundation for Stakeholder Involvement Approaches to Evaluation (A Response to Cousins, Whitmore, and Shulha, 2013). Am J Eval. 2014;35:144-8

37. Arnstein SR. A Ladder of Citizen Participation. J Am Plan Assoc. 2019;85:24-34.

38. Schram JL, Oude Groeniger J, Schuring M, et al. Working conditions and health behavior as causes of educational inequalities in self-rated health: an inverse odds weighting approach. Scand J Work Environ Health. 2020;76:0-9.

39. Dieker ACM, ljzelenberg W, Proper Kl, et al. The contribution of work and lifestyle factors to socioeconomic inequalities in self-rated health - a systematic review. Scand J Work Environ Heal. 2019;45:114-25.

40. Karpowitz CF, Raphael C, Hammond AS IV. Deliberative democracy and inequality: Two cheers for enclave deliberation among the disempowered. Polit Soc. 2009:37:576-615.

41. Nierse CJ, Abma TA. Developing voice and empowerment: The first step towards a broad consultation in research agenda setting. J Intellect Disabil Res. 2011;55:411-21.

42. Abma TA WG. Responsieve methodologie. Interactief onderzoek in de praktijk. 1st ed. Den Haag: Uitgeverij LEMMA, 2006.

43. Pini B. Interviewing men: Gender and the collection and interpretation of qualitative data. J Sociol. 2005;41:201-16.

44. Verdonk P, Seesing H, De Rijk A. Doing masculinity, not doing health? a qualitative study among dutch male employees about health beliefs and workplace physical activity. BMC Public Health. 2010;10:712.

\section{Publisher's Note}

Springer Nature remains neutral with regard to jurisdictional claims in published maps and institutional affiliations.

Ready to submit your research? Choose BMC and benefit from:

- fast, convenient online submission

- thorough peer review by experienced researchers in your field

- rapid publication on acceptance

- support for research data, including large and complex data types

- gold Open Access which fosters wider collaboration and increased citations

- maximum visibility for your research: over 100M website views per year

At BMC, research is always in progress.

Learn more biomedcentral.com/submissions 